\title{
Reproductibilité de l'observation de la muqueuse buccale en autofluorescence pour le diagnostic de lésions à potentiel malin
}

\section{Catros S, Glock N, Lérici S, Gaston R, Meddeb M, Savel H, Baschet L, Doussau A, Fricain JC (Pôle d'Odontologie et de Santé Buccale, CHU de Bordeaux)}

Afin d'améliorer le dépistage précoce des lésions à potentiel malin et cancéreuses de la muqueuse buccale, l'examen en autofluorescence a été proposé en complément de l'examen conventionnel en lumière incandescente. En effet, les lésions dysplasiques et cancéreuses de la muqueuse buccale peuvent être mises en évidence par un examen en autofluorescence (Lane 2006). Des lésions infra-cliniques peuvent être révélées par l'autofluorescence (Poh 2007).

L'objectif principal était d'évaluer la reproductibilité inter-observateur (junior/senior) de l'examen de la cavité buccale en autofluorescence $(\mathrm{AF})$ tissulaire, en situation de dépistage des lésions à potentiel malin, des dysplasies et des cancers de la muqueuse buccale, dans une population de patients alcoolo-tabagiques. L'objectif secondaire visait à évaluer l'apport potentiel de l'AF par rapport à un examen visuel simple par lumière incandescente (LI) dans le dépistage des mêmes lésions dans la même population. II s'agissait d'une étude diagnostique transversale, appariée, avec évaluation en insu, sur une population de patients consécutifs.

Les critères d'inclusion étaient un âge supérieur à 18 ans, une consommation d'alcool supérieure à 14 verres pour les femmes et 21 verres pour les hommes, un tabagisme actif ou sevré depuis moins d'un an. Les critères d'exclusion étaient la présence d'une pathologie de l'hémostase sévère et la présence d'une pathologie précancéreuse, dysplasique ou cancéreuse connue de la muqueuse buccale.

Le principal critère d'évaluation était la concordance inter-observateur (junior/senior), vis-à-vis de la décision de biopsier une lésion à potentiel pré malin, une dysplasie ou un cancer après examen en LI puis AF. La concordance inter observateur a été mesurée par le coefficient kappa de cohen. 180 patients ont été inclus dans cette étude.

A la fin de l'étude, les praticiens juniors et seniors avaient observé 589 et 484 anomalies en LI et 572 et 476 anomalies en AF, respectivement. Une indication de biopsie a été posée en LI chez 42 et 39 patients pour les juniors et les seniors respectivement. En AF, l'indication de biopsie a été posée chez 45 et 47 patients pour les juniors et les seniors respectivement. 31 biopsies ont été réalisées. Elles ont mis en évidence 2 dysplasies modérées, 1 carcinome épidermoïde micro-invasif et 2 carcinomes épidermoïdes invasifs. Le coefficient simple kappa vis à vis de la décision de biopsier n'était pas significativement supérieur à 0,6 en LI et était supérieur à 0,6 Iorsque l'on rajoutait la fluorescence. Concernant l'évaluation de l'efficacité, l'AF a permis de détecter une lésion supplémentaire chez les juniors par rapport à l'examen en LI.

L'ajout de la fluorescence à l'examen clinique a significativement augmenté la concordance junior/senior vis à vis de la décision de biopsier une lésion à potentiel malin dans une population de patients alcoolo-tabagique. Ce résultat suggère que la fluorescence peut être utilisée en situation de dépistage par les juniors et les seniors.

CATROS Sylvain

sylvain.catros@u-bordeaux2.fr

This is an Open Access article distributed under the terms of the Creative Commons Attribution License 2.0, which permits unrestricted use, distribution, and reproduction in any medium, provided the original work is properly cited. 